\title{
TÉCNICAS DE INTELIGÊNCIA ARTIFICIAL APLICADAS AOS PROCESSOS DE GESTÃO HOSPITALAR
}

\author{
Carlos Roberto Franzini Filho (UAM) crfilho@anhembi.br \\ Adiloderne Nogueira Souza Filho (UAM) anfilho@anhembi.br
}

\section{Resumo}

Um hospital pode absorver um alto nível de inovação tecnológica e ser qualificado como "de ponta" ou não a partir do nível de integração dos sistemas de informação com a tecnologia hospitalar presente. Os hospitais apresentam-se como organizações complexas que envolvem um grande número relevante de processos administrativos e apresentam altos custos em razão da sua natureza. A partir desta abordagem, observa-se uma série de desafios e oportunidades a serem enfrentados pelos profissionais da área da engenharia na melhoria contínua dos processos a partir da adoção de técnicas de inteligência artificial, incluindo os hospitais. A Inteligência Artificial (IA) pode ser definida como a ciência e engenharia capaz de extrair a capacidade das máquinas em simular o pensamento dos seres humanos, obtendo a capacidade de aprender, raciocinar, perceber, deliberar e decidir de forma racional e inteligente a respeito de um determinado problema e a utilização de algoritmos pode contribuir para a tomada de decisões adequadas e fornecer alertas simultaneamente a todas as áreas e subáreas dentro de um hospital. A partir deste cenário, este trabalho propõe-se a apresentar uma revisão bibliográfica sobre técnicas de inteligência artificial aplicadas aos processos de gestão hospitalar. Espera-se que as informações obtidas a partir da revisão bibliográfica realizada contribua como incentivo para futuras pesquisas e fomente o interesse dos docentes em engenharia em ministrar tais conteúdos em suas aulas. Sugere-se o aprofundamento de pesquisas nas subáreas que abrangem a inteligência artificial.

Palavras-Chaves: Inteligência Artificial, Gestão Hospitalar, Revisão Bibliográfica. 


\section{Introdução}

Um hospital pode absorver um alto nível de inovação tecnológica e ser qualificado como "de ponta" ou não a partir do nível de integração dos sistemas de informação com a tecnologia hospitalar presente (SALU, 2013). A necessidade de acesso à informação é cada vez maior por parte dos envolvidos no setor de saúde, desde o usuário, quanto ao conhecimento da situação de sua saúde e decisão sobre os procedimentos, pelos profissionais da saúde, quanto à informação clínica presente quando e onde necessária, até gestores que necessitam de dados para tomada de decisão, por exemplo, em relação ao monitoramento da qualidade dos cuidados disponibilizados (SOUSA, 2012).

Segundo a Organização Mundial da Saúde (OMS) a função de um hospital é ofertar assistência completa, tanto curativa quanto preventiva aos seus pacientes, tendo uma atuação extensiva à família, sendo um centro de formação para os profissionais da área e suas pesquisas biossociais. Para Borba e Lisboa (2006) uma estrutura hospitalar, conforme é por demais complexa, envolve vários profissionais especialistas. Os hospitais apresentam-se como organizações complexas que envolvem um grande número relevante de processos administrativos e apresentam altos custos em razão da sua natureza e características e para o estabelecimento de uma estrutura onde se faz necessário o agrupamento dentro de funções que permitam a formação de departamentos, serviços, setores harmônicos entre si, constituindo uma organização lógica e racional do hospital (OLIVEIRA e TODA2013).

Os conceitos aplicados à serviços, devem ser aplicados a todas as organizações que prestam algum tipo de serviço, tal como hospitais e podem ser estendidos aos hospitais (FITZSIMMONS e FITZSIMMONS, 2014). Simultaneidade é um conceito sobre administração de serviços que não podem ser estocados, produção e consumo acontecem de forma simultânea. Perecibilidade de um serviço é a capacidade ociosa perdida por falta de demanda que em serviço não pode ser estocado. Serviços são conceitos e ideias não patenteáveis, assim, aplica-se o conceito de intangibilidade. A heterogeneidade ocorre em serviços, pois, a variação do serviço prestado muda de cliente para cliente, em hospitais cada paciente recebe um atendimento único. Segundo Cavallini e Bisson (2010) o hospital deve ser considerado como uma empresa e como tal pode ser classificado em função do tamanho (pequena, média ou grande), tipo de atividade (produção de bens ou serviços), forma de propriedade (pública ou privada) e tipo de controle (coercitiva, utilitária ou normativa).

A Inteligência Artificial (IA) pode ser definida como a ciência e engenharia capaz de extrair a capacidade das máquinas em simular o pensamento dos seres humanos, obtendo a capacidade 
de aprender, raciocinar, perceber, deliberar e decidir de forma racional e inteligente a respeito de um determinado problema (MC CARTHY, 1958). A utilização de algoritmos pode contribuir para a tomada de decisões adequadas e fornecer alertas simultaneamente a todas as áreas e subáreas de um hospital. Para a adoção da IA nos processos hospitalares, observa-se a necessidade de: disponibilidade de um grande volume de dados, programação de algoritmos treinados e testados com técnicas de aprendizado de máquina, em uma infraestrutura de Tecnologia da Informação (TI) automatizada e com alta capacidade de processamento computacional (VAZ, 2017). Um sistema de IA consegue analisar dados em grande escala, identificar padrões e tendências e, então, formular predições de forma automática com velocidade e precisão. Nesse contexto, percebe-se diversas possibilidades de aplicações de IA para empresas e também órgãos públicos incluindo os hospitais. Para Arariboia (1988), as técnicas de IA imitam mecanismos da natureza por intermédio de mecanismos tecnológicos cujo desenvolvimento foi baseado em mecanismos naturais. Dentre as principais técnicas de IA consideradas para a realização deste estudo estão: bayesian network, clustering, data mining, deep learning, expert system, fuzzy, image analysis, neural networks, random forest e support e vector machine.

A partir das considerações iniciais, observa-se uma série de desafios e oportunidades a serem enfrentados pelos profissionais da área da engenharia no Brasil em um futuro próximo na melhoria contínua dos processos hospitalares a partir da adoção de técnicas de inteligência artificial. Dentro desta abordagem, este trabalho propõe-se a apresentar uma revisão bibliográfica sobre técnicas de inteligência artificial aplicadas em processos de gestão hospitalar.

\section{Referencial teórico}

A inovação tecnológica como fator chave para obter vantagem competitiva está entre as principais características de uma prestação de serviços, e conforme Pinto, Nossa e Teixeira (2015), a recente crise mundial evidenciou a necessidade de uma gestão diferenciada, eficiente, de forma a manter o hospital como negócio rentável, aplicando a redução de custos e aumento da produtividade, de forma a garantir retorno sobre os investimentos realizados, sendo a inovação fator chave para obter estes quesitos.

Para Akenroye (2012), a rápida mutação em relação ao quadro médico dos pacientes, o envelhecimento da população e a necessidade de redução de custos levam à necessidade de 
avanços tecnológicos constantes. Neste contexto, observa-se a inteligência artificial (IA) definida como um ramo da ciência e engenharia capaz de analisar dados complexos e seu potencial para explorar uma relação significativa com um conjunto de dados para ser usado no diagnóstico, tratamento e previsão de resultados em diferentes cenários nos processos hospitalares (SHAPIRO, 1992). Existem muitas aplicações bem-sucedidas de IA nas áreas de gestão de processos hospitalares. Para Strickland (2014), os modelos preditivos são os algoritmos para a implementação de modelos estatísticos relacionados às distribuições de probabilidade, ou a modelos de regressão de dados e estatísticas relacionadas, ou a modelos matemáticos teóricos, ou a modelos de redes neurais artificiais, ou modelos de gráficos probabilísticos. As principais técnicas de IA consideradas para a realização deste estudo estão apresentadas no Quadro 1: bayesian network, clustering, data mining, deep learning, expert system, fuzzy, image analysis, neural networks, random forest e support e vector machine.

Quadro 1 - Técnicas de inteligência artificial (IA).

\begin{tabular}{|c|c|c|}
\hline \multicolumn{2}{|r|}{ Técnica } & Definição/aplicação \\
\hline 1. & $\begin{array}{l}\text { Rede bayesiana ou } \\
\text { "bayesian network" }\end{array}$ & $\begin{array}{l}\text { Matematicamente uma rede bayesiana é uma representação compacta de uma } \\
\text { tabela de conjunção de probabilidades do universo do problema. Do ponto de } \\
\text { vista de um especialista, redes bayesianas constituem um modelo gráfico que } \\
\text { representa de forma simples as relações de causalidade das variáveis de um } \\
\text { sistema (KORB e NICHOLSON, 2010). A técnica de IA rede bayesiana } \\
\text { apresenta-se como uma técnica promissora para os processos de gestão } \\
\text { hospitalar em razão da capacidade de analisar sistemas complexos e suas inter- } \\
\text { relações. }\end{array}$ \\
\hline 2. & Clustering & $\begin{array}{l}\text { A técnica de "clustering" que consiste em um agrupamento automático de } \\
\text { dados segundo a sua semelhança segundo Strickland (2014). }\end{array}$ \\
\hline 3. & $\begin{array}{l}\text { Mineração dos dados } \\
\text { ou "data mining" }\end{array}$ & $\begin{array}{l}\text { Data mining ou mineração de dados é a técnica de IA que consiste na } \\
\text { organização } \quad \text { dos } \\
\text { padrões, associações, mudanças e/ou anomalias relevantes. A aplicação da } \\
\text { mineração de dados se dá em grandes bases de dados contidos dentro do data } \\
\text { warehouse e pode contribuir com a tomada de decisão pelas instituições de } \\
\text { forma mais rápida e com maior grau de confiança (CARDOSO e MACHADO, } \\
\text { 2008). }\end{array}$ \\
\hline 4. & $\begin{array}{l}\text { Aprendizagem } \\
\text { Profunda ou } \\
\text { "deep learning" }\end{array}$ & $\begin{array}{l}\text { A técnica de "deep learning" ou aprendizagem profunda refere-se ao número } \\
\text { de camadas através das quais os dados são transformados, onde cada camada } \\
\text { sucessiva usa a camada anterior como entrada formando uma hierarquia de } \\
\text { conceitos, } 2014 \text { (DENG et al., 2014). }\end{array}$ \\
\hline 5. & $\begin{array}{l}\text { Sistemas especialistas } \\
\text { ou expert system }\end{array}$ & $\begin{array}{l}\text { Os sistemas especialistas ou "expert system" são programas com o objetivo de } \\
\text { simular o raciocínio de um profissional "expert" em alguma área específica do } \\
\text { conhecimento (HJERPPE e OLANDER, 1989) }\end{array}$ \\
\hline 6. & $\begin{array}{l}\text { Lógica difusa } \\
\text { ou "fuzzy logic", }\end{array}$ & $\begin{array}{l}\text { A lógica difusa ou "fuzzy logic" é a técnica de IA empregada para descrever a } \\
\text { inter-relação entre os processos hospitalares e graduar a sua relevância na qual } \\
\text { os valores lógicos das variáveis podem ser qualquer número real entre } 0 \text {, } \\
\text { correspondente ao valor falso e } 1 \text {, correspondente ao valor verdadeiro. A lógica } \\
\text { difusa lida com o conceito de verdade parcial, onde o valor verdade pode } \\
\text { compreender entre o valor completamente verdadeiro e completamente falso } \\
\text { (GOMIDE e GUDWIN, 1994). }\end{array}$ \\
\hline 7. & $\begin{array}{l}\text { Análise de imagens ou } \\
\text { "image analysis" }\end{array}$ & $\begin{array}{l}\text { A análise de imagem ou "image analysis" é a técnica de IA que estuda } \\
\text { automaticamente uma imagem com o objetivo de extrair informações úteis e } \\
\text { relevantes a partir dela. Para este a composição deste trabalho, não foram }\end{array}$ \\
\hline
\end{tabular}




\begin{tabular}{|l|l|l|}
\hline & & $\begin{array}{l}\text { identificados artigos dentro do escopo na literatura. Sua aplicação concentra-se } \\
\text { nos processos de diagnósticos diversos (SOLOMON e BRECKON, 2011). }\end{array}$ \\
\hline 8. & $\begin{array}{c}\text { Aprendizado de } \\
\text { máquina ou } \\
\text { "machine learning" }\end{array}$ & $\begin{array}{l}\text { A técnica "machine learning" ou aprendizado de máquina é a técnica de IA } \\
\text { onde o aprendizado automático da máquina sem ser programada explora o } \\
\text { estudo e a construção de algoritmos capazes de aprender com seus erros e fazer } \\
\text { previsão sobre dados. Tais algoritmos operam construindo um modelo a partir } \\
\text { de inputs amostrais a fim de fazer previsões ou decisões guiadas pelos dados ao } \\
\text { invés de simplesmente seguindo inflexíveis e estáticas instruções programadas } \\
\text { (MONARD e BARANAUSKAS, 2003). }\end{array}$ \\
\hline 9. & $\begin{array}{l}\text { Redes Neurais ou } \\
\text { "neural networks" }\end{array}$ & $\begin{array}{l}\text { Redes neurais artificiais (RNAs) ou "neural network" é a técnica de IA } \\
\text { inspirada no sistema nervoso central de um animal (em particular o cérebro) } \\
\text { que são capazes de realizar o aprendizado de máquina bem como } \\
\text { o reconhecimento de padrões. Redes neurais artificias geralmente são } \\
\text { apresentadas como sistemas de "neurônios interconectados, que podem } \\
\text { computar valores de entradas", simulando o comportamento de redes neurais } \\
\text { biológicas (VAZ, 2019). }\end{array}$ \\
\hline 10. & $\begin{array}{l}\text { Floresta aleatória ou } \\
\text { "random forest" }\end{array}$ & $\begin{array}{l}\text { A técnica de IA random forest ou floresta aleatória é um algoritmo de } \\
\text { aprendizagem supervisionada que cria múltiplas árvores de decisão que podem } \\
\text { ser utilizadas para tanto para tarefas de classificação quanto para regressão (HO, } \\
\text { 1995). }\end{array}$ \\
\hline 11. & $\begin{array}{l}\text { Máquina de vetores de } \\
\text { suporte } \\
\text { ou support vector machine (SVM) ou máquina de vetores de suporte é a técnica } \\
\text { "support vector } \\
\text { machine" }\end{array}$ & $\begin{array}{l}\text { IA para um conjunto de métodos do aprendizado supervisionado que } \\
\text { analisam os dados e reconhecem padrões usados para classificação e análise de } \\
\text { regressão. O SVM padrão toma como entrada um conjunto de dados e prediz, } \\
\text { para cada entrada dada, qual de duas possíveis classes a entrada faz parte, o que } \\
\text { faz do SVM um classificador linear binário não probabilístico (CORTES e } \\
\text { VAPNIK, 1995). }\end{array}$ \\
\hline
\end{tabular}

Fonte: Elaborado pelos autores (2019).

\section{Metodologia}

A partir da realização de uma revisão narrativa da literatura, buscou-se apresentar discussões conceituais sobre o tema técnicas de IA aplicadas em processos de gestão hospitalar. A "revisão narrativa" não aplica critérios explícitos e sistemáticos na busca e análise crítica da literatura. A seleção dos estudos e interpretação das informações podem estar sujeitas à subjetividade dos autores e este tipo de revisão não necessita esgotar as fontes de coleta de informações (UNESP, 2015).

A coleta de dados englobou periódicos nacionais e internacionais disponíveis nas bases de dados disponibilizadas pela Universidade Anhembi Morumbi (UAM): CAPES, EBSCO, PUBMED, WEB OF SCIENCE e GOOGLE ACADÊMICO. As buscas foram concentradas nas plataformas a partir das palavras chaves:

$$
\begin{aligned}
& \text { "hospital" + "bayesian network"; } \\
& \text { "hospital" + “clustering”; } \\
& \text { "hospital" + “data mining"; }
\end{aligned}
$$


"hospital" + "deep learning”;

"hospital" + "expert system";

"hospital" + "fuzzy";

"hospital" + "image analysis";

"hospital" + "machine learning";

"hospital" + "neural networks";

"hospital" + "random forest";

"hospital" + "support vector machine".

Para os critérios de inclusão e exclusão de artigos, este estudo buscou identificar exclusivamente as publicações relacionadas às técnicas de IA aplicadas a processos de gestão hospitalar e não abrange as técnicas de IA aplicadas a outros temas da medicina e/ou saúde como por exemplo, o diagnóstico e tratamento de doenças e/ou desenvolvimento de fármacos.

A partir da adoção da IA nos processos hospitalares, os tomadores de decisão dispõem de melhores informações que podem levar à melhorias de qualidade, maior satisfação dos clientes/pacientes, minimização de horas-extras, redução de custos, maiores receitas, melhor alocação dos recursos, redução do tempo de espera dos pacientes pelos serviços hospitalares e redução de exposição a riscos.

\section{Análise e discussão dos resultados}

As técnicas de IA são recomendadas para lidar com tarefas de tomada de decisão envolvidas no gerenciamento de organizações complexas como os hospitais. A adoção de técnicas de IA nos processos de gestão hospitalar podem desempenhar um papel fundamental nos processos de planejamento estratégico dos hospitais, permitindo a integração de aplicativos, bancos de dados, softwares e hardwares essenciais aos usuários, o que viabiliza a análise em tempo real de um grande volume de informações complexas e contribui de forma relevante na otimização da tom ada de decisões para os processos. De acordo com os critérios de inclusão e exclusão de artigos propostos na metodologia, a Tabela 1 apresenta os resultados a partir das buscas realizadas nas plataformas disponibilizadas pela UAM para as técnicas de IA consideradas para a realização deste trabalho. 
Tabela 1 - Volume de artigos incluídos e excluídos para o estudo.

\begin{tabular}{|c|c|c|c|c|}
\hline \multicolumn{2}{|r|}{ Palavras-Chave } & \multirow{2}{*}{$\begin{array}{c}\begin{array}{c}\text { Artigos } \\
\text { Encontrados }\end{array} \\
169\end{array}$} & \multirow{2}{*}{$\begin{array}{c}\begin{array}{c}\text { Artigos } \\
\text { Considerados }\end{array} \\
22\end{array}$} & \multirow{2}{*}{$\begin{array}{c}\begin{array}{c}\text { de } \\
\text { aproveitamento }\end{array} \\
13 \% \\
\end{array}$} \\
\hline 1. & "hospital" + "bayesian network" & & & \\
\hline 2. & "hospital" + “clustering" & 86 & 4 & $4,7 \%$ \\
\hline 3. & "hospital" + "data mining" & 77 & 15 & $19,5 \%$ \\
\hline 4. & "hospital" + "deep learning" & 25 & 3 & $12 \%$ \\
\hline 5. & "hospital" + "expert system" & 20 & 6 & $30 \%$ \\
\hline 6. & "hospital" + "fuzzy" & 100 & 43 & $43 \%$ \\
\hline 7. & "hospital" + "image analysis" & 39 & 0 & $0 \%$ \\
\hline 8. & "hospital" + "machine learning" & 140 & 16 & $11,4 \%$ \\
\hline 9 & "hospital" + "neural networks" & 42 & 3 & $7,1 \%$ \\
\hline 10. & "hospital" + "random forest"; & 16 & 2 & $12,5 \%$ \\
\hline 11. & "hospital" + "support vector machine". & 8 & 1 & $12,5 \%$ \\
\hline
\end{tabular}

Fonte: Elaborado pelos autores (2019).

A Figura 1 apresenta a análise prévia para a quantidade total de 722 artigos encontrados nas plataformas de dados disponibilizadas pela UAM sobre as técnicas de IA aplicadas aos processos de gestão hospitalar”. Foram descartados 611 artigos em razão de estarem repetidos e/ou fora do escopo previsto para a realização deste trabalho. Dessa forma, observa-se um total de 111 artigos selecionados pelo autor para a realização deste estudo.

Figura 1 - Análise prévia dos artigos encontrados.

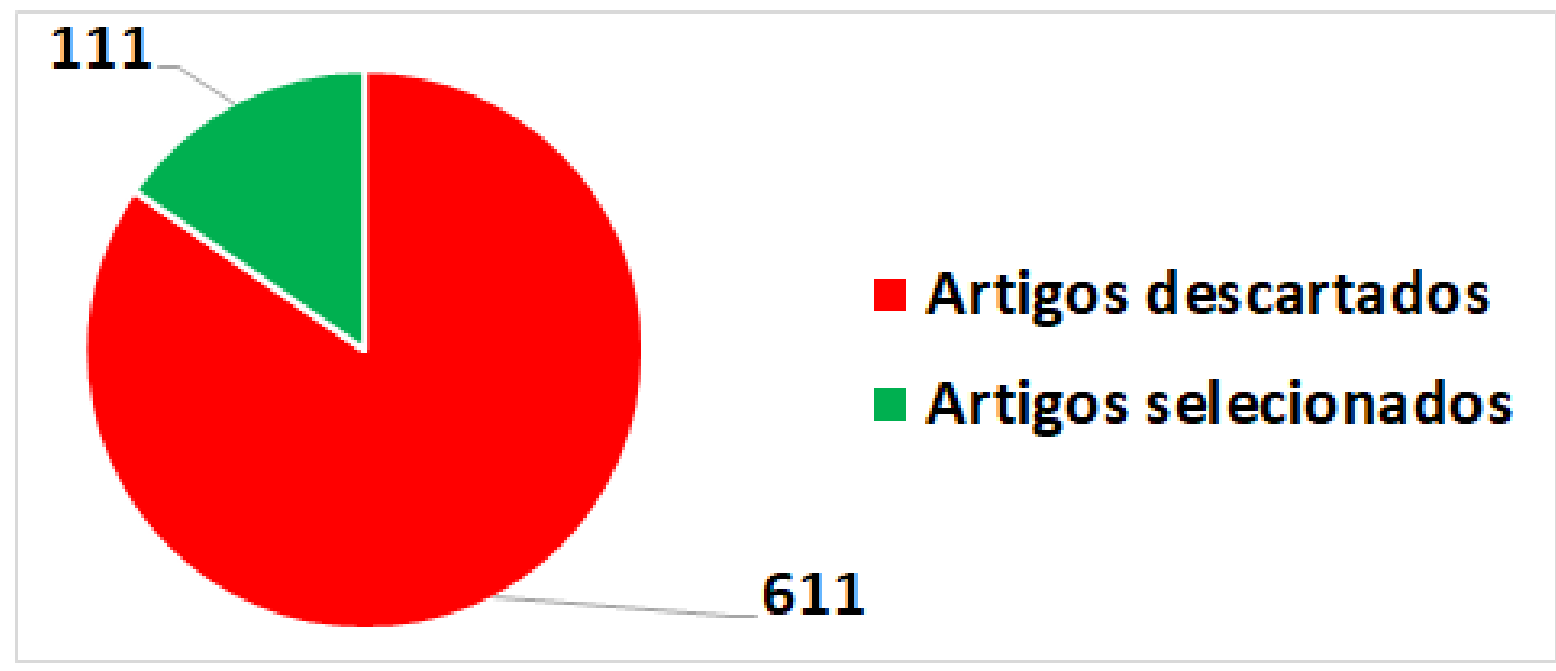

Fonte: Elaborado pelos autores (2019).

A Figura 2 ilustra em ordem decrescente a quantidade de artigos selecionados de acordo as técnicas de IA aplicadas aos processos de gestão hospitalar". Observa-se que a técnica de IA mais aplicada aos processos se gestão hospitalar de acordo com os selecionados é a técnica de lógica difusa $(37,4 \%)$, seguida da rede bayesiana $(19,1 \%)$, machine learning $(13,4 \%)$ e mineração dos dados $(13,4 \%)$. 
Figura 2 - Análise prévia dos artigos encontrados X técnica de IA.

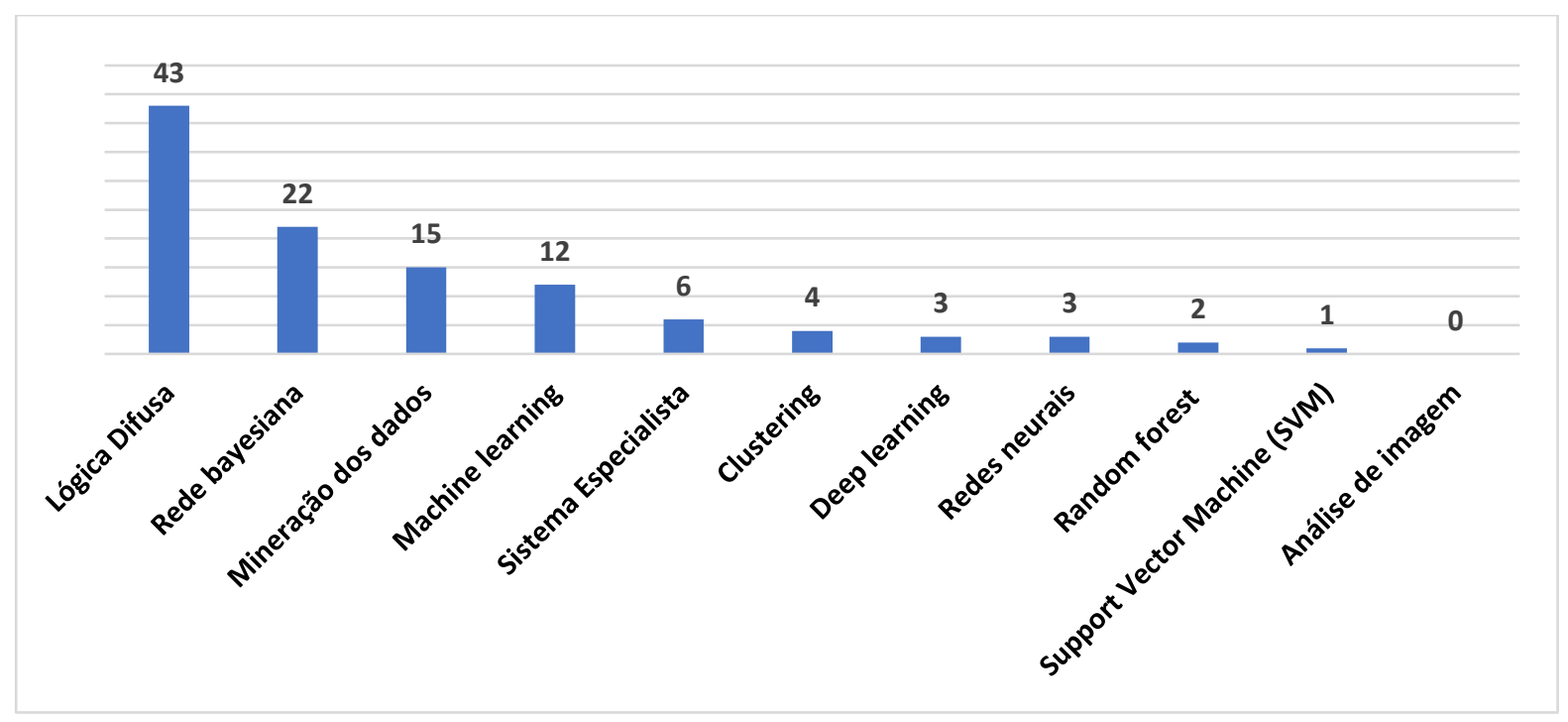

Fonte: Elaborado pelo autor (2019).

O Quadro 2 ilustra uma matriz das técnicas de IA X processos de gestão hospitalar aplicáveis. Observa-se nesta matriz os processos hospitalares com mais aplicações as técnicas de IA consideradas para a realização deste trabalho: gestão de custos hospitalares, fluxo de pacientes, investimentos em infraestrutura, eficiência hospitalar, previsão de ocupação de leitos (internações e re-internações), gestão da qualidade e processos de triagem de pacientes.

Quadro 2 - Matriz das técnicas de IA X processos de gestão hospitalar.

\begin{tabular}{|c|c|c|c|c|c|c|c|c|c|c|c|c|}
\hline \multirow{2}{*}{\multicolumn{2}{|c|}{$\begin{array}{l}\text { Principais áreas e processos hospitalares onde são } \\
\text { aplicadas/estudadas as técnicas de inteligência } \\
\text { artificial }\end{array}$}} & \multicolumn{11}{|c|}{ Técnicas de IA } \\
\hline & & \multirow{2}{*}{ 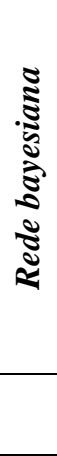 } & \multirow{2}{*}{ } & \multirow{2}{*}{ 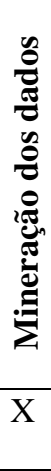 } & \multirow{2}{*}{ 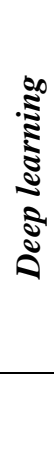 } & \multirow{2}{*}{ 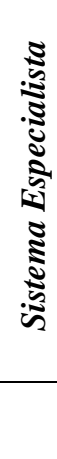 } & \multirow{2}{*}{ 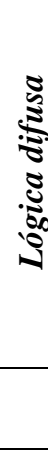 } & \multirow{2}{*}{ 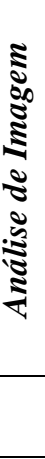 } & \multirow{2}{*}{ 离 } & \multirow{2}{*}{ 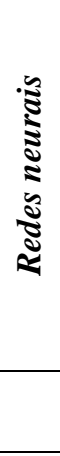 } & \multirow{2}{*}{ 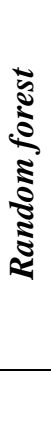 } & \multirow{2}{*}{ 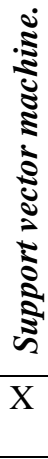 } \\
\hline 1. & $\begin{array}{c}\text { Agendamento de consultas, exames e } \\
\text { cirurgias. }\end{array}$ & & & & & & & & & & & \\
\hline 2. & $\begin{array}{l}\text { Análise de desempenho médico e serviços } \\
\text { hospitalares. }\end{array}$ & $\mathrm{X}$ & & & & & & & & & & \\
\hline 3. & $\begin{array}{l}\text { Controle de medicamentos e insumos } \\
\text { hospitalares. }\end{array}$ & & & & & $\mathrm{X}$ & & & & & & \\
\hline 4. & Fluxo de pacientes. & $\mathrm{X}$ & & & & & & & & $\mathrm{X}$ & $\mathrm{X}$ & $\mathrm{X}$ \\
\hline 5. & Eficiência hospitalar. & $\mathrm{X}$ & $\mathrm{X}$ & & & $\mathrm{X}$ & & & & $\mathrm{X}$ & & \\
\hline 6. & Escala de profissionais da saúde. & & & & & & & & & & & \\
\hline 7. & Estratégias preventivas de saúde. & $\mathrm{X}$ & $\mathrm{X}$ & $\mathrm{X}$ & & & & & & & & \\
\hline 8. & Gerenciamento de ativos. & & & & & & & & & & $\mathrm{X}$ & \\
\hline 9. & Gestão da Qualidade Total (TQM). & $\mathrm{X}$ & & $\mathrm{X}$ & & & & & & & & \\
\hline 10 & Gestão de custos hospitalares. & $\mathrm{X}$ & & & $\mathrm{X}$ & $\mathrm{X}$ & & & & $\mathrm{X}$ & & \\
\hline 11. & Gestão de risco financeiro & & & $\mathrm{X}$ & & & & & & & & \\
\hline 12. & Integração/alocação de recursos. & $\mathrm{X}$ & $\mathrm{X}$ & & & & & & & & & \\
\hline 13. & Investimentos em infraestrutura. & & & & $\mathrm{X}$ & & & & & $X$ & & $X$ \\
\hline
\end{tabular}




\begin{tabular}{|c|c|c|c|c|c|c|c|c|c|c|c|c|}
\hline 14. & Layout de instalações. & & & $\mathrm{X}$ & & $\mathrm{X}$ & & & & \\
\hline 15. & Modelagem de centros cirúrgicos. & & & & & & & & & & & \\
\hline 16. & Previsão de ocupação de leitos (internações). & $\mathrm{X}$ & & & $\mathrm{X}$ & & $\mathrm{X}$ & & $\mathrm{X}$ & & & \\
\hline 17. & Procedimentos cirúrgicos. & $\mathrm{X}$ & & & & & & & & & & \\
\hline 18. & Processos de capacitação e treinamento. & $\mathrm{X}$ & & & & & & & & & & \\
\hline 19. & Processos de triagem de pacientes. & & & $\mathrm{X}$ & & $\mathrm{X}$ & $\mathrm{X}$ & & & $\mathrm{X}$ & & \\
\hline 20. & Recrutamento de profissionais da saúde. & $\mathrm{X}$ & & & & & & & & & & \\
\hline 21. & Registros hospitalares. & & $\mathrm{X}$ & & & & $\mathrm{X}$ & & & & & \\
\hline 22. & $\begin{array}{c}\text { Riscos de contaminação pela exposição dos } \\
\text { servidores da saúde. }\end{array}$ & $\mathrm{X}$ & & & & & & & & $\mathrm{X}$ & & \\
\hline
\end{tabular}

Fonte: Elaborado pelo autor (2019).

\section{Considerações finais}

O objetivo do presente trabalho foi realizar uma revisão bibliográfica narrativa sobre as técnicas de inteligência artificial (IA) aplicadas aos processos de gestão hospitalar. Como resultado desta análise, observa-se as como técnicas de IA mais promissoras a lógica difusa, rede bayesiana, mineração dos dados e machine learning.

A IA é uma técnica recomendada pelos autores e periódicos identificados na realização deste trabalho para lidar com as tarefas de tomada de decisão envolvidas no gerenciamento de organizações complexas como os hospitais. A adoção da IA nos processos hospitalares pode desempenhar um papel fundamental nos processos de planejamento estratégico dos hospitais, permitindo a integração de aplicativos, bancos de dados, softwares e hardwares essenciais aos usuários, o que viabiliza a análise em tempo real de um grande volume de informações complexas e contribui de forma relevante na otimização da tom ada de decisões para os processos.

As limitações para a realização desta pesquisa apresentam-se com relação a identificação exclusiva de publicações relacionadas às técnicas de inteligência artificial aplicada aos processos de gestão hospitalar e não abrange a aplicação de técnicas de inteligência artificial aplicadas a outros temas da medicina e/ou saúde como por exemplo o tratamento de doenças e/ou desenvolvimento de fármacos.

Diante da relevância do tema proposto para a realização deste trabalho, tanto para os hospitais quanto para o meio acadêmico, espera-se que as informações obtidas a partir da realização deste trabalho contribua com incentivo para futuras pesquisas e fomente o interesse dos hospitais na adoção da inteligência artificial em seus processos. Neste contexto, sugere-se o aprofundamento de pesquisas em diferentes subáreas abrangidas pela inteligência artificial e processos hospitalares específicos identificados a partir da realização deste trabalho. Também se 
recomenda o aprofundamento desta pesquisa em técnicas especificas de aplicação da IA e aplicações em outros processos que não sejam voltados a gestão hospitalar.

É importante destacar que boa parte dos hospitais brasileiros ainda possuem os registros médicos dos seus pacientes em papel, o que impede o compartilhamento de informações e consequentemente inibe o desenvolvimento do sistema de saúde com aplicação da IA.

\section{Agradecimentos}

Os agradecimentos à Universidade Anhembi Morumbi pelo apoio e incentivo a pesquisa, seu corpo docente, administração e coordenação do curso de Engenharia de Produção.

\section{REFERÊNCIAS}

AKENROYE, T. O. Factors influencing innovation in healthcare: a conceptual synthesis. The Innovation Journal, v. 17, n. 2, p. 1, 2012.

ARARIBOIA, G. Inteligência Artificial. Rio de Janeiro: Ed. LTC, 1988.

BORBA, V. R.; LISBOA, T. C. Teoria Geral de Administração Hospitalar: Estrutura e Evolução do processo de gestão hospitalar. Rio de Janeiro: Ed. Qualitymark, 2006.

CARDOSO O.N.P., MACHADO R.T.M. Gestão do conhecimento usando data mining: estudo de caso na Universidade Federal de Lavras. Rev Adm Pública. 2008;42(3):495-528.

CAVALLINI, M. E.; BISSON, M. P. Farmácia Hospitalar: um enfoque em sistemas de saúde. 2. ed. São Paulo: Ed. Manole, 2010, 304 p.

CORTES, Corinna; VAPNIK, Vladimir. Support-vector networks. Machine learning, v. 20, n. 3, p. 273-297, 1995.

DENG, Li et al. Deep learning: methods and applications. Foundations and Trends® in Signal Processing, v. 7, n. 3-4, p. 197-387, 2014.

Faculdade de Ciências Agronômicas - UNESP Campus de Botucatu. Tipos de revisão da literatura. Disponível em: 〈http://www.fca.unesp.br/Home/Biblioteca/tipos-de-evisao-de-literatura.pdf >. Acesso em: 24 mar. 2019.

FITZSIMMONS, J. A.; FITZSIMMONS, M. J. Administração de Serviços: Operações, Estratégia e Tecnologia da Informação. AMGH Editora, 2014.

GOMIDE, Fernando Antonio Campos; GUDWIN, Ricardo Ribeiro. Modelagem, controle, sistemas e lógica fuzzy. SBA controle \& Automação, v. 4, n. 3, p. 97-115, 1994.

HJERPPE, Roland; OLANDER, Birgitta. Cataloging and expert systems: AACR2 as a knowledge base. Journal of the American Society for Information Science, v. 40, n. 1, p. 27-44, 1989.

HO, Tin Kam. Random decision forests. In: Proceedings of 3rd international conference on document analysis and recognition. IEEE, 1995. p. 278-282.

KORB, Kevin B.; NICHOLSON, Ann E. Bayesian artificial intelligence. CRC press, 2010. 
MCCARTHY, John. Programs with common sense, paper presented at the Symposium on the Mechanization of Thought Processes, National Physical Laboratory, Teddington, England, Nov. 24-27, 1958. (Published in Proceedings of the Symposium).

MONARD, Maria Carolina; BARANAUSKAS, José Augusto. Conceitos sobre aprendizado de máquina. Sistemas inteligentes-Fundamentos e aplicações, v. 1, n. 1, p. 1, 2003.

SALU, E. J. Administração hospitalar no Brasil. Barueri, SP: Ed. Manole, 2013.

SOLOMON, Chris; BRECKON, Toby. Fundamentals of Digital Image Processing: A practical approach with examples in Matlab. John Wiley \& Sons, 2011.

SOUSA, P. Sistemas de Informação em Enfermagem: novos desafios, novas oportunidades. Revista da Escola de Enfermagem da USP. v. 46, n. 5, p. 1-2, out. 2012.

SHAPIRO, Stuart C. Encyclopedia of artificial intelligence second edition. John, 1992.

STRICKLAND, Jeffrey. Predictive modeling and analytics. Lulu. com, 2014.

VAZ, Tiago Andres. Modelo de dados para treinamento de inteligência artificial na pesquisa em saúde: um estudo prático sobre infecções hospitalares. 2017. Disponível em: 〈https://www.lume.ufrgs.br/handle/10183/181275>. Acesso em: 15 fev. 2019. 